\title{
mtDNA copy number associated with age of onset in familial amyloid polyneuropathy
}

\author{
Diana Santos, ${ }^{1,2,3}$ Maria João Santos, ${ }^{4,5}$ Miguel Alves-Ferreira, ${ }^{1,2,3}$ Teresa Coelho, ${ }^{6}$ \\ Jorge Sequeiros, ${ }_{1}^{1,2,3,7}$ Isabel Alonso, 1,2,7,3 Pedro Oliveira, ${ }^{3,8}$ Alda Sousa, 1,2,3 \\ Carolina Lemos, ${ }^{1,2,3}$ Manuela Grazina ${ }^{4,5}$
}

- Additional material is

published online only. To view please visit the journal online (http://dx.doi.org/10.1136/ jnnp-2017-316657)

${ }^{1}$ i3S, Instituto de Investigação e Inovação em Saúde,

Universidade do Porto, Porto, Portugal

${ }^{2}$ UnIGENe, Instituto de Biologia Molecular e Celular (IBMC),

Porto, Portugal

${ }^{3}$ Instituto Ciências Biomédicas Abel Salazar (ICBAS),

Universidade do Porto, Porto, Portugal

${ }^{4}$ Centre for Neuroscience and Cell Biology, Laboratory of Biochemical Genetics (LGB), Universidade de Coimbra,

Coimbra, Portugal

${ }^{5}$ Faculdade de Medicina da Universidade de Coimbra (FMUC), Coimbra, Portugal ${ }^{6}$ Unidade Corino de Andrade (UCA), Centro Hospitalar do Porto (CHP), Porto, Portugal

${ }^{7}$ Centro de Genética Preditiva e Preventiva (CGPP), Instituto de Biologia Molecular e Celular (IBMC) and Instituto de Investigação e Inovação em Saúde (i3S), Universidade do

Porto, Porto, Portugal

${ }^{8}$ Instituto de Saúde Pública (ISPUP), Universidade do Porto Porto, Portugal

Correspondence to Dr Carolina Lemos, ICBAS, UnIGENe, IBMC and i3S, University Porto, Rua Alfredo Allen, n 208, 4200-135 Porto, Portugal; clclemos@ibmc.up.pt

$\mathrm{CL}$ and $\mathrm{MG}$ contributed equally.

Received 13 June 2017 Revised 8 September 2017 Accepted 25 September 2017 Published Online First 10 October 2017

Check for updates

To cite: Santos D, Santos MJ, Alves-Ferreira M, et al.J Neurol Neurosurg Psychiatry 2018:89:300-304.

\section{ABSTRACT}

Background Transthyretin-related familial amyloid polyneuropathy (TTR-FAP Val30Met) shows a wide variation in age-at-onset $(\mathrm{AO})$ between generations and genders, as in Portuguese families, where women display a later onset and a larger anticipation (>10 years). Mitochondrial DNA (mtDNA) copy number was assessed to clarify whether it has a modifier effect on $A 0$ variability in Portuguese patients.

Methods The mtDNA copy number of 262 samples (175 Val30Met TTR carriers and 87 controls (proven Val30Val)) was quantified by quantitative real-time PCR. Statistical analysis was performed using IBM SPSS V.23 software.

Results This study shows that Val30Met TTR carriers have a significantly higher $(p<0.001)$ mean mtDNA copy number than controls. Furthermore, the highest mtDNA copy number mean was observed in early-onset patients (AO <40 years). Importantly, early-onset offspring showed a significant increase $(p=0.002)$ in the mtDNA copy number, when compared with their late $\mathrm{AO}$ parents. Conclusions The present findings suggest, for the first time, that mtDNA copy number may be associated with earlier events and may therefore be further explored as a potential biomarker for follow-up of TTR-FAP Val30Met carriers.

\section{INTRODUCTION}

Transthyretin-related familial amyloid polyneuropathy (TTR-FAP) is an autosomal dominant systemic amyloidosis due to point mutations in the TTR gene (chr18q12.1) (Online Mendelian Inheritance in Man, OMIM,176300), resulting in misfolded proteins. Although more than 100 amyloidogenic variants have been found in the TTR gene, ${ }^{1}$ Val30Met (also known as NM_000371.3:c.148G>Ap. (Val50Met)) is the most common.

FAP was classified by Andrade $^{2}$ as a peculiar form of neuropathy and was first described in northern Portugal as a disease occurring between 25 and 35 years. However, differences in mean age-at-onset (AO) between clusters have been described, including within the Portuguese population. ${ }^{3-5}$ In TTR-FAP Val30Met Portuguese families, a wide AO variability (19-82 years) and AO differences between generations and genders have been observed. ${ }^{4}$

Recently, our group showed that anticipation (a decrease in AO over the generations) is a true biological phenomenon in TTR-FAP Val30Met. ${ }^{6}$
Moreover, clinical differences between early-onset ( $<40$ years) and late-onset ( $\geq 50$ years) patients have also been described in other countries. ${ }^{7-10}$ Additionally, in Portuguese patients, significant differences in AO regarding gender were confirmed, namely that women present a later-onset than men and larger anticipation ( $>10$ years) was more frequent when the disease was inherited from the mothers $(70 \%)$ than from the fathers (30\%). In addition, mother-son pairs showed a larger anticipation, while father-daughter pairs showed only a residual anticipation. ${ }^{6}$ To clarify gender-related differences, we previously reported, for the first time, the contribution of the $A R$ gene as an AO modifier in both genders. ${ }^{11}$

The mitochondrial DNA (mtDNA) is inherited exclusively from the mother, ${ }^{12}$ and in contrast to fixed diploid nuclear genome, mitochondria are polyploids, that is, have hundreds to several thousand copies of mtDNA for each cell, depending on the energy demands of the tissue or the developmental stage. ${ }^{13}$

The regulation of mtDNA copy number is an important aspect of mitochondrial genetics and biogenesis, essential for normal cellular function and crucial for maintaining cellular energy requirements. Thus, depletion, variation, decrease or excess of mtDNA copy number may be associated with several neurodegenerative diseases. ${ }^{14-16}$

A previous study showed that an mtDNA variant may explain the observed differences in penetrance for TTR-FAP according to the transmitting parent gender in Portuguese families, ${ }^{17}$ and that mtDNA haplogroups may be associated with AO variation in TTR-FAP Swedish and French patients. ${ }^{18}$ However, the analysis of mtDNA copy number was never performed in TTR-FAP Val30Met before; therefore, we believe that this is a groundbreaking approach.

To further explore the remarkable $\mathrm{AO}$ variation between genders and especially due to the anticipation when the mother is the transmitting parent, our aim was to evaluate, for the first time, whether the mtDNA copy number has a modifier effect in TTR-FAP Val30Met families.

\section{SUBJECTS AND METHODS \\ Subjects}

DNA samples were ascertained from Unidade Corino de Andrade-Centro Hospitalar do Porto (UCA-CHP, Porto), which has the largest database 
Table 1 Sample description of Val30Met TTR carriers and non-carriers

\begin{tabular}{|c|c|c|c|c|c|c|}
\hline \multirow{2}{*}{$\begin{array}{l}\text { Status } \\
\text { Characteristics }\end{array}$} & \multicolumn{3}{|c|}{ Val30Met $T T R$ carriers $(\mathrm{n}=175)$} & \multicolumn{3}{|c|}{ Non-carriers $(n=87)$} \\
\hline & Early-onset & Late-onset & Asymptomatic & Relatives & Controls & Total \\
\hline $\mathrm{N}$ & 56 & 52 & 67 & 30 & 57 & 262 \\
\hline Age-at-onset, years & $(24-39)$ & $(40-71)$ & na & na & na & na \\
\hline Age-at-observation, years & na & na & $(19-81)$ & $(18-76)$ & $(21-89)$ & na \\
\hline \multicolumn{7}{|l|}{ Gender } \\
\hline Male & 27 & 21 & 27 & 15 & 29 & 119 \\
\hline Female & 29 & 31 & 40 & 15 & 28 & 143 \\
\hline
\end{tabular}

na, not applicable; $T T R$, transthyretin.

of TTR-FAP Val30Met worldwide, with a registry collected and clinically well-characterised over 75 years. All participants signed a written informed consent. All carriers have the Val30Met disease-causing variant, and the AO of each patient has been established by the same team of neurologists, specialised in TTR-FAP Val30Met, when the first neuropathic symptoms (sensorimotor or autonomic) emerged. In the smaller number of cases where cardiac or kidney involvement is the sole symptom, their manifestation also defines onset of the disease.

We have analysed a total of 262 blood-derived DNA samples from 56 early-onset ( $<40$ years) and 52 late-onset ( $\geq 40$ years) patients, 67 asymptomatic carriers (aged $\geq 40$ years) and 30 non-carriers (proven Val30 Val $(\mathrm{V} 30 \mathrm{~V})$ relatives belonging to the same familial background). Control subjects (proven V30V) included blood donors, and patients' spouses ( $\mathrm{n}=57 ; 29$ men and 28 women), without any TTR-FAP Val30Met familial history, were also enrolled in this study (table 1). DNA was isolated by the same method for all selected subjects. Additionally, all samples were genotyped for Val30Met disease-causing variant and matched for the same ethnic and geographical origin of Portugal. All DNA samples were collected and stored at the Centro de Genética Preditiva e Preventiva Biobank, authorised by National Commission for Data Protection.

\section{DNA extraction}

Genomic DNA was extracted from peripheral blood leucocytes using a standard salting out method, ${ }^{19}$ according to the manufacturer's instructions. Details on the method for DNA quantification are presented as online supplementary material 1.

\section{mtDNA extraction}

The relative mtDNA copy number was measured in all samples using quantitative real-time PCR. This method for determining mtDNA copy number was detailed in a previous publication by Venegas and Halberg ${ }^{20}$ and was shown to have high interassay reliability. Details on the method for determining mtDNA copy number are presented as online supplementary material 1.

\section{Statistical analysis}

Sample size was estimated and is in accordance with the expected power.

Multiple comparisons among groups were analysed by one-way analysis of variance (one-way ANOVA) followed by post-hoc corrections with Tukey HSD (honest significant difference) method.

In order to investigate the effect of mtDNA copy number in $\mathrm{AO}$ variation, a generalised estimating equations (GEE) analysis, to account for non-independence of AO between members of the same family, was performed, adjusted for gender. Evaluation of the parent-offspring transmissions was achieved using the t-test for paired samples. All statistical procedures were performed using the transformed variable for mtDNA copy number except for the initial descriptive analysis. The level of significance for all statistical tests was set at $\mathrm{p}<0.05$. All statistical analyses were performed using IBM SPSS Statistics V.23 software. More details on statistical analysis are presented in online supplementary material 1.

\section{RESULTS}

In table 2, we can find the descriptive analysis for mean mtDNA copy number for all groups studied, comprising 175 Val30Met TTR carriers and 87 non-carriers, in a total sample of 262 individuals of Portuguese origin. It is important to note that the non-transformed mean mtDNA copy number of non-carriers $(155.21 \pm 85.23)$ was lower than that of Val30Met TTR carriers $(460.11 \pm 478.91)$.

\section{mtDNA copy number of peripheral blood leucocytes is} associated with Val30Met disease-causing variant carriers Normality of our sample was tested and the Kolmogorov-Smirnov test showed that the data were not normally distributed $(\mathrm{p}<0.05)$.

We investigated differences in mean mtDNA copy number (using the transformed variable) and we found a statistically significant difference between groups as shown by the one-way ANOVA test $(F(4,257)=6.611 ; \mathrm{p}<0.001)$. A Tukey post-hoc test revealed that the mtDNA copy number in controls was significantly lower, compared with asymptomatic carriers $(p<0.001)$, late-onset patients $(p=0.001)$ and early-onset patients $(\mathrm{p}<0.001)$. There were no statistically significant differences between the controls and non-carrier relatives $(p>0.05)$, neither between non-carrier relatives and Val30Met carriers (p>0.05) (figure 1).

Table 2 Descriptive analysis of mean mtDNA copy number for all the groups studied

\begin{tabular}{llclr}
\hline & & \multicolumn{3}{c}{ Mean mtDNA copy } \\
Status & Groups & $\mathbf{n}$ & number/cell & \multicolumn{1}{c}{ SD } \\
\hline Non-carriers & Controls & 57 & 140.19 & 63.11 \\
& Relatives & 30 & 183.75 & 112.09 \\
& All & 87 & 155.21 & 85.23 \\
Val30Met & Asymptomatic & 67 & 406.58 & 382.37 \\
TTR carriers & Late-onset & 52 & 479.74 & 527.21 \\
& Early-onset & 56 & 505.92 & 535.17 \\
& All & 175 & 460.11 & 478.91 \\
\hline
\end{tabular}

mtDNA, mitochondrial DNA; TTR, transthyretin. 


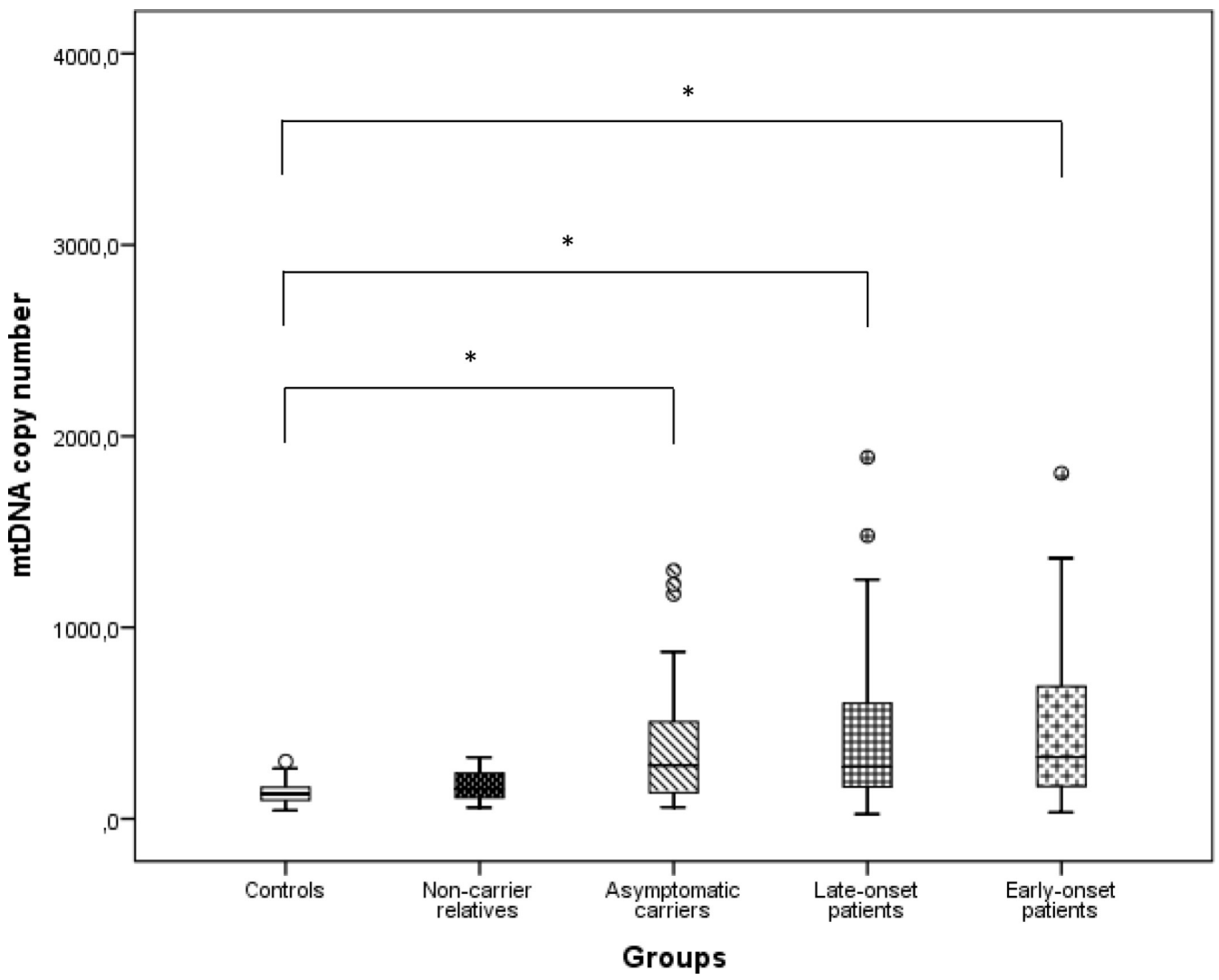

Figure 1 Analysis of differences in mtDNA copy number for various groups in study (non-carriers: controls and relatives; and Val30Met TTR carriers: asymptomatic carriers, late-onset patients and early-onset patients). Asterisks $\left(^{*}\right)$ indicate statistical significance, $p<0.05$. mtDNA, mitochondrial DNA; TTR, transthyretin.

Concerning gender differences, no significant differences associated to mtDNA copy number were found in all groups studied ( $p>0.05$; results not shown).

\section{mtDNA copy number is not associated with $\mathrm{AO}$ variation}

The role of the mtDNA copy number on AO variation between the late-onset and early-onset patients, using GEE, did not show a significant effect ( $p>0.05$; results not shown).

\section{Variation of mtDNA content in the parent-offspring transmissions}

Regarding parent-offspring pairs analysis, no significant difference was found between asymptomatic carriers with their affected parents $(p>0.05)$, even when stratified by AO of the affected parents. Importantly, early-onset offspring showed a statistically significant increase in the mtDNA copy number, when compared with their late AO parents $(\mathrm{p}=0.002)$ (table 3$)$.

\section{DISCUSSION}

In the present study, mtDNA copy number was quantified in TTR-FAP Val30Met carriers and non-carriers to better explore the $\mathrm{AO}$ variation and its effect on gender of the transmitting parent and offspring, since to date a minor attention has been dedicated to mtDNA content in this disease. To our knowledge, this is the first study that demonstrates a positive association between the peripheral blood mtDNA content and the TTR-FAP Val30Met, with implications in the underlying biological mechanisms.

\section{Increased mtDNA copy number in Val30Met disease-causing variant carriers}

Comparing the different groups, we have found that the highest mean mtDNA copy number per cell was observed in early-onset patients. Moreover, subjects harbouring the Val30Met diseasecausing variant have higher mean mtDNA content than controls. Our results contrast with studies in other diseases, such as in Huntington's $^{21}$ and Parkinson's ${ }^{22}$ diseases, in which the mtDNA copy number per cell declined in variant carriers compared with controls, possibly revealing a compensatory effect.

A previous study showed that increased cellular mtDNA copy number and mtDNA variants accumulated during ageing may be caused by oxidative stress. ${ }^{23}$ It is plausible that the increase

Table 3 Analysis of mitochondrial DNA copy number, using the transformed variable, for parent-offspring transmissions stratified by age-at-onset of the affected parents

\begin{tabular}{lllllll}
\hline Affected parent & Offspring & $\mathbf{n}$ & Mean & SD & 95\% Cl & p Value* \\
\hline Late-onset & Asymptomatic & 26 & -162.41 & 508.82 & {$[-367.93$ to 43.10] } & 0.116 \\
Early-onset & Asymptomatic & 18 & -149.25 & 462.48 & {$[-379.23$ to 80.73] } & 0.189 \\
\hline Late-onset & Early-onset & 14 & -307.11 & 293.63 & {$[-476.64$ to 137.57] } & 0.002 \\
\hline *Significance level was set to 0.05. & & & & & \\
\hline 302 & & & & & & \\
\hline
\end{tabular}


in mitochondrial content in Val30Met carriers, particularly in early-onset patients, may be due to a compensatory response to maintain mitochondrial function possibly owing to decreased cytochrome c-oxidase (complex IV) function, as observed in blood cells from patients with amyotrophic lateral sclerosis. ${ }^{24}$ Also, previous reports lead us to suggest that increased levels of mtDNA content, mainly in Val30Met carriers, may be related with higher production of reactive oxidative species (ROS), possibly due to the unpaired cellular processes, such as oxidative stress and the presence of misfolded proteins, occurring in TTR-FAP Val30Met progression. ${ }^{25}{ }^{26}$ It is well known that the functions of mitochondria and endoplasmic reticulum (ER) are closely linked, since both produce ROS, generated by products of oxidative phosphorylation ${ }^{27}$ and by unfolded-protein response. ${ }^{28}{ }^{29}$ Indeed, several studies have described that high levels of ROS may disrupt protein folding processes, and increased production of misfolded proteins results in extra ER stress due to the accumulation of misfolded proteins, possibly leading to additional ROS production. ${ }^{29}$ For that reason, we hypothesised that an adaptation of the energetic metabolism, to improve mitochondrial function and cell growth ${ }^{30}$ must have occurred in these patients through a compensatory mechanism, involving an increased ROS production and, consequently, energy failure. Therefore, to our knowledge, the present findings revealed, for the first time, that mtDNA copy number is associated with earlier TTR-FAP Val30Met events.

Moreover, we found significant differences between controls and Val30Met carriers, suggesting that the mtDNA content is associated with the aetiopathogenic mechanisms of the disease. Interestingly, the mtDNA content is not significantly different neither between controls and non-carrier relatives nor between non-carrier relatives and Val30Met carriers, which can be due to a common genetic background between relatives of the same families besides the Val30Met disease-causing variant, showing that the genetic background is an important issue in studying the genetic modifiers of patients' phenotype.

\section{mtDNA content has no gender-linked effect}

Regarding gender analysis, these results show that mtDNA content does not have a specific gender-linked effect similar to what was observed in other diseases, ${ }^{3132}$ or that it is under the control of nuclear genes, inherited from either the mother or the father.

\section{Early-onset offspring have higher mtDNA copy number than their affected parents}

The comparison of mtDNA content of late-onset offspring with their affected or non-affected parents could not be performed due to the small sample size of the parents group. Importantly, a higher mean mtDNA copy number was found in the earlyonset offspring when compared with their late-onset parents. This result suggests that early-onset offspring possibly receive from their affected mother a mitochondrial risk effect, modulating $\mathrm{AO}$.

In conclusion, this study suggests that mtDNA copy number seems to have a significant effect on $\mathrm{AO}$ variability observed in parent-offspring transmission. In addition, we found that mitochondrial gene expression is possibly associated with TTR-FAP Val30Met mechanisms. However, the mtDNA content is specific for tissue and developmental stage/age and varies according to the population. Therefore, it will be important to replicate this study in other tissues, such as liver (main organ of TTR synthesis), and in different populations, to unravel new clues about the biological mechanisms that explain the role of mitochondria energetic performance in processing of misfolded proteins.

Our results, although preliminary, derive from the largest TTR-FAP Val30Met cohort available worldwide. In addition, we had a special concern with the power of the statistical analyses to obtain accurate and reliable results.

Although further studies are required to elucidate the pathophysiological significance of the observed changes in mitochondrial content in patients with TTR-FAP Val30Met, the present study is a step forward and opens a new possibility for elucidating the mechanisms underlying TTR-FAP Val30Met. mtDNA copy number may also be further explored as a potential biomarker for follow-up of TTR-FAP Val30Met carriers.

Acknowledgements We would like to thank all patients for participating in this study, and Vanessa Costa for all the help in assembling family data. In addition, we would like to thank FEDER funds through the Programa Operacional Factores de Competitividade - COMPETE 2020, and by national funds through the FCT - Fundação para a Ciência e a Tecnologia (COMPETE: POCI-01-0145FEDER-007440). This work was supported by grants from Fundação para a Ciência e Tecnologia, FCT (PTDC/SAU-GMG/100240/2008 and PEsT), cofunded by ERDF and COMPETE, and by Financiamento Plurianual de Unidades de Investigação (FCT).

Contributors Conception and design of the study: AS, CL, MG. Acquisition and analysis of data: TC, DS, MA-F, PO, AS, CL. Drafting a significant portion of the manuscript or figures: DS, MJS, AS, CL, MG. Critical revision of the manuscript for important intellectual content: MJS, TC, MA-F, PO, JS, IA, AS. Statistical expertise: PO, $A S, C L$. Obtaining funding: TC, IS, IA, AS, CL. Administrative, technical or material support: MA-F, IA, CL. Study supervision: AS, CL, MG.

Funding DS and MA-F are recipients of an FCT fellowship (SFRH/BD /91160/2012 and SFRH/BD/101352/2014, respectively). Our funding sources supported the data collection and analysis, but did not play a role in the study design, in interpretation of data, in the writing of the report or in the decision to submit the paper for publication.

Competing interests DS has received research support from an FCT fellowship (SFRH/BD /91160/2012). TC's institution has received support from FoldRx Pharmaceuticals, which was acquired by Pfizer in October 2010; TC has served on the scientific advisory board of Pfizer and received funding from Pfizer for scientific meeting expenses (travel, accommodations and registration). She currently serves on the THAOS (natural history disease registry) scientific advisory board. MA-F has received research support from an FCT fellowship (SFRH/BD/101352/2014). MJS, JS, $\mathrm{PO}, I \mathrm{~A}, \mathrm{AS}, \mathrm{CL}$ and $\mathrm{MG}$ report no disclosures.

Ethics approval The Ethics Committee of Hospital de Santo António (HAS-CHP, Porto) approved the study.

Provenance and peer review Not commissioned; externally peer reviewed.

(c) Article author(s) (or their employer(s) unless otherwise stated in the text of the article) 2018. All rights reserved. No commercial use is permitted unless otherwise expressly granted.

\section{REFERENCES}

1 Benson MD. Pathogenesis of transthyretin amyloidosis. Amyloid 2012;19(Suppl 1):14-15.

2 Andrade C. A peculiar form of peripheral neuropathy; familiar atypical generalized amyloidosis with special involvement of the peripheral nerves. Brain 1952;75:408-27.

3 Sousa A, Andersson R, Drugge U, et al. Familial amyloidotic polyneuropathy in Sweden: geographical distribution, age of onset, and prevalence. Hum Hered 1993:43:288-94

4 Sousa A, Coelho T, Barros J, et al. Genetic epidemiology of familial amyloidotic polyneuropathy (FAP)-type I in Póvoa do Varzim and Vila do Conde (north of Portugal). Am J Med Genet 1995;60:512-21.

5 Ikeda S, Nakazato M, Ando Y, et al. Familial transthyretin-type amyloid polyneuropathy in Japan: clinical and genetic heterogeneity. Neurology 2002;58:1001-7.

6 Lemos C, Coelho T, Alves-Ferreira M, et al. Overcoming artefact: anticipation in 284 Portuguese kindreds with familial amyloid polyneuropathy (FAP) ATTRV30M. J Neurol Neurosurg Psychiatry 2014;85:326-30.

7 Koike H, Misu K, Sugiura M, et al. Pathology of early- vs late-onset TTR Met30 familial amyloid polyneuropathy. Neurology 2004;63:129-38.

8 Ihse $E$, Ybo A, Suhr $\mathrm{O}$, et al. Amyloid fibril composition is related to the phenotype of hereditary transthyretin V30M amyloidosis. J Pathol 2008;216:253-61.

9 Buades-Reinés J, Raya-Cruz M, Gallego-Lezaún C, et al. Transthyretin familial amyloid polyneuropathy (TTR-FAP) in Mallorca: a comparison between late- and early-onset disease. J Peripher Nerv Syst 2016;21:352-6. 
10 Koike H, Ikeda S, Takahashi M, et al. Schwann cell and endothelial cell damage in transthyretin familial amyloid polyneuropathy. Neurology 2016;87:2220-9.

11 Santos D, Coelho T, Alves-Ferreira M, et al. Variants in RBP4 and AR genes modulate age at onset in familial amyloid polyneuropathy (FAP ATTRV30M). Eur J Hum Genet 2015.

12 Schon EA. Mitochondrial genetics and disease. Trends Biochem Sci 2000;25:555-60.

13 Schon EA, DiMauro S, Hirano M. Human mitochondrial DNA: roles of inherited and somatic mutations. Nat Rev Genet 2012;13:878-90.

14 Blokhin A, Vyshkina T, Komoly S, et al. Variations in mitochondrial DNA copy numbers in MS brains. J Mol Neurosci 2008;35:283-7.

15 Morten KJ, Ashley N, Wijburg F, et al. Liver mtDNA content increases during development: a comparison of methods and the importance of age- and tissue-specific controls for the diagnosis of mtDNA depletion. Mitochondrion 2007; 7:386-95.

16 Bai RK, Wong LJ. Simultaneous detection and quantification of mitochondrial DNA deletion(s), depletion, and over-replication in patients with mitochondrial disease. J Mol Diagn 2005;7:613-22.

17 Bonaïti B, Olsson M, Hellman U, et al. TTR familial amyloid polyneuropathy: does a mitochondrial polymorphism entirely explain the parent-of-origin difference in penetrance? Eur J Hum Genet 2010;18:948-52.

18 Olsson M, Hellman U, Planté-Bordeneuve V, et al. Mitochondrial haplogroup is associated with the phenotype of familial amyloidosis with polyneuropathy in Swedish and French patients. Clin Genet 2009;75:163-8.

19 Miller SA, Dykes DD, Polesky HF. A simple salting out procedure for extracting DNA from human nucleated cells. Nucleic Acids Res 1988;16:1215.

20 Venegas V, Halberg MC. Measurement of mitochondrial DNA copy number. Methods Mol Biol 2012;837:327-35.
21 Petersen MH, Budtz-Jørgensen E, Sørensen SA, et al. Reduction in mitochondrial DNA copy number in peripheral leukocytes after onset of Huntington's disease. Mitochondrion 2014;17:14-21.

22 Pyle A, Anugrha H, Kurzawa-Akanbi M, et al. Reduced mitochondrial DNA copy number is a biomarker of parkinson's disease. Neurobiol Aging 2016;38:216-e7-10.

23 Lee $\mathrm{HC}$, Yin PH, Lu CY, et al. Increase of mitochondria and mitochondrial DNA in response to oxidative stress in human cells. Biochem J 2000;348(Pt 2):425-32.

24 Ehinger JK, Morota S, Hansson MJ, et al. Mitochondrial dysfunction in blood cells from amyotrophic lateral sclerosis patients. J Neurol 2015;262:1493-503.

25 Ando Y, Nyhlin N, Suhr O, et al. Oxidative stress is found in amyloid deposits in systemic amyloidosis. Biochem Biophys Res Commun 1997;232:497-502.

26 Hou X, Aguilar MI, Small DH. Transthyretin and familial amyloidotic polyneuropathy. Recent progress in understanding the molecular mechanism of neurodegeneration. Febs J 2007;274:1637-50.

27 Dröge W. Free radicals in the physiological control of cell function. Physiol Rev 2002;82:47-95.

28 Görlach A, Klappa P, Kietzmann T. The endoplasmic reticulum: folding, calcium homeostasis, signaling, and redox control. Antioxid Redox Signal 2006;8(9-10):1391-418.

29 Haynes CM, Titus EA, Cooper AA. Degradation of misfolded proteins prevents ERderived oxidative stress and cell death. Mol Cell 2004;15:767-76.

30 Jeng JY, Yeh TS, Lee JW, et al. Maintenance of mitochondrial DNA copy number and expression are essential for preservation of mitochondrial function and cell growth. $J$ Cell Biochem 2008;103:347-57.

31 He Y, Tang J, Li Z, et al. Leukocyte mitochondrial DNA copy number in blood is not associated with major depressive disorder in young adults. PLoS One 2014;9:e96869.

32 Zhang Y, Qu Y, Gao K, et al. High copy number of mitochondrial DNA (mtDNA) predicts good prognosis in glioma patients. Am J Cancer Res 2015;5:1207-16. 


\section{NNP mtDNA copy number associated with age of onset in familial amyloid polyneuropathy}

Diana Santos, Maria João Santos, Miguel Alves-Ferreira, Teresa Coelho, Jorge Sequeiros, Isabel Alonso, Pedro Oliveira, Alda Sousa, Carolina Lemos and Manuela Grazina

J Neurol Neurosurg Psychiatry2018 89: 300-304 originally published online October 10, 2017

doi: 10.1136/jnnp-2017-316657

Updated information and services can be found at:

http://jnnp.bmj.com/content/89/3/300

\section{These include:}

References This article cites 31 articles, 2 of which you can access for free at: http://jnnp.bmj.com/content/89/3/300\#ref-list-1

Email alerting service

Receive free email alerts when new articles cite this article. Sign up in the box at the top right corner of the online article.

\section{Notes}

To request permissions go to:

http://group.bmj.com/group/rights-licensing/permissions

To order reprints go to:

http://journals.bmj.com/cgi/reprintform

To subscribe to BMJ go to:

http://group.bmj.com/subscribe/ 